\title{
Sexo, género, sexualidad. La pertinencia de un enfoque constructivista*
}

\section{Raquel Osborne}

UNED. Facultad de Ciencias Politicas y Sociología. Deparcamento de Sociología II c/ Senda del Rey, s/n. 28044 Madrid. Spain

\section{Resumen}

Al igual que "el género" es una categoría sociaimente construida, necesitamos de un enfoque constructivista para el análisis de la sexualidad, análisis que aquí nos interesa en la medida en que afecta a la situación de subordinación femenina. Si la sexualidad no responde a nada esencial ni natural sino que se halla histórica, cultural y socialmente construida, y si además la analizamos cn un contexto de dominación/subordinación, la consecuencia será que podemos cambiar esas pautas, pero para ello necesitamos, entre orros factores, mucha más investigación y rigor académicos a la hora de tratar esta temática.

Palabras clave: sexo, género, sexualidad, constructivismo, feminismo.

Abstract. Sex, gender, sexuality. A constructionist approach

A construc gender is a social construction. A constructionist analysis of sexuality is needed to understand woman's subordination. Sexuality is a historical cultural and social construction in a domination versus subordination context. (Redaction)

Key words: sex, gender, sexuality, constructionism, feminism.

Si tenemos en cuenta el título de esta mesa, afortunadamente podemos decir que a estas alturas ya no podemos considerar al género como una categoría nueva a la hora de analizar la realidad social. Desde principios de los años setenta, sociólogas norteamericanas del prestigio de Jessie Bernard o Arlie Hochschild, entre otras, criticaton el sesgo masculino tanto en la teoría como en la metodología de las ciencias sociales, y utilizaron como eje de su crítica lo que tradicionalmente se inclúa bajo la denominación de "roles sexuales».

* Este trabajo fue presentado como ponencia en la sesión " $\mathrm{E}$ l género como una nueva categoría para el análisis de la realidad social?» incluida en el simposium Sociología del género. IV Congreso español de sociología. Madrid, 24 al 26 de septiembre de 1992. El tono coloquial se ha conservado en cierta medida, aunque con retoques. 
Desde entonces ha llovido bastante en todas partes, aunque en España todavía reste por consolidar una tendencia y un camino abiertos ya por muchas mujeres de dentro y de fuera de la Academia, entre otras las aquí presentes. Y si digo que resta por consolidar es porque una cosa es el trabajo de crítica realizado por parte de quienes defienden la necesaria inclusión de una perspectiva de género en el análisis de la realidad social, y otra algo distinta es su aceptación por parte de la comunidad sociológica en general. Desde luego que la inserción de una sesión como ésta en el presente congreso atestigua que se ha recorrido un largo trecho. No es cuestión, no obstante, con todo y ser importante, de estudiar sólo "el tema de la mujer» o incluir la variable «sexo» a fin de analizar situaciones que nos conciernen y que hasta hace poco no se tenían en cuenta. Se trata de utilizar un enfoque feminista en las ciencias sociales, en este caso la sociología, que no otra cosa supone hablar de una perspectiva de género. Entendemos por género el constructo social que convierte a hombres y mujeres en seres sociales frente a su concepción como meras entidades biológicas, seres que por lo demás se hallan encuadrados en unas determinadas relaciones de dominación/subordinación.

Yo por mi parte querría ceñirme más al papel de la sexualidad en la desigualdad social por razón de sexo y a la forma en que podemos tratar esta temática en las ciencias sociales. La única matización que añadiré a lo ya dicho es que si tradicionalmente ha costado, y seguixá costando, la aceptación del enfoque ferninista en las ciencias sociales, mencionar expresamente la dimensión de la sexualidad desde esta óptica puede plantear todavía algunas resistencias, bien por considerársele un tema al que aún rodea un cierto tabú y al que nos acercamos con una media sonrisa, bien por concederle poca importancia y ser visto meramente como un epifenómeno a la hora de analizar la discriminación por razón de sexo.

Lo primero que hemos de tener en cuenta es que, al igual que la categoría de «género» responde a lo que socialmente llegamos a ser partiendo de la materia prima biológica que en origen nos conforma en tanto que seres humanos, según lo que acabamos de comentar, ta sexualidad, tal y como la vivimos, la entendemos, la gozamos y la sufrimos, no responde para nada a una supuesta libido natural, como desde hace más de un siglo nos han hecho creer la medicina, la psicología, la psiquiatría y la sexología. Experimentamos la sexualidad a nivel individual o personal, pero a la hora de estudiarla y entenderla podemos afirmar, con Gayle Rubin, que los deseos se hallan constituidos en el curso de prácticas históricas y sociales específicas (Rubin, 1984: 276). Los vivimos individualmente a través de nuestros cuerpos, pero también nttestros propios cuerpos poseen un significado cultural, no natural (ibidem: 276-277). Podemos destacar, así, que no hay una esencia acerca de qué es lo que constituye la sexualidad, no hay nada inmutable al respecto.

Que veamos la sexualidad como un hecho no natural, es decir, histórico y sociocultural, nos lleva a romper con la idea de que existe un sustrato sexual natural en los seres humanos. Con enfoques de este tipo se rompe con las teorías de la sexualidad que tradicionalmente han dominado el panorama y que afir- 
man, a) o bien que el sexo es algo fundamentalmente peligroso, que por tanto sólo puede ser aceptado si se canaliza en forma apropiada hacia el matrimonio, o b) que el sexo es básicamente saludable y bueno, sólo que ha sido reprimido y negado por una sociedad corrupta.

Estamos aludiendo implfcitamente a la denuncia de la "hipótesis represiva» que formuló Michel Foucault en su Historia de la sexualidad y que tanto ha contribuido, entre otras cosas, a desmirificar una era como la victoriana. Dicha época, en contra de lo que comúnmente se creía, no representó meramente la represión de la sexualidad, sino que comportó una amplia producción, categorización y multiplicación de los discursos sobre la sexualidad. Es lo que Foucautt denomina "el dispositivo de la sexualidad".

Abandonar la hipótesis represiva nos sirve, además, como señala Jeffrey Weeks, para intentar comprender los múltiples mecanismos del poder en un momento histórico determinado, mecanismos que no son dirigidos por una sola entidad o grupo sino que responden a complejos procesos de relaciones $e$ intereses. El poder no se ejerce de una forma monolítica, sino que podríamos decir "que se va ejerciendo" (Schur, 1980: 7), que es un proceso que da lugar a mecanismos contradictorios, mecanismos que producen al mismo tiempo la dominación y la oposición a esa dominación, la subordinación y la resistencia (Weeks, 1986: 36-37). De ahí que, tampoco en el caso de las mujeres, sea correcto imaginar que todo ha sido mera represión, con todo y lo mucho que nuestra sexualidad nos ha sido negada. Cabe pensar más bien que, en un contexto de dominación que ha tenido lugar desde hace mucho tiempo y de muy diversas maneras, ha habido lugar para oposiciones y resistencias. La propia existencia del feminismo da testimonio de ello.

Las preocupaciones feministas en torno a la sexualidad provienen ya del primer feminismo organizado en el siglo XIX, preocupaciones que denunciaban cómo las diferencias de sexo se utilizaban para establecer relaciones de dominación de los hombres sobre las mujeres, los problemas de explotación sexual, o las posibilidades de consenso y elección en este terreno (Weeks, 1986: 89). Posteriormente, ya en los años veinte y treinta del presente siglo, tras el surgimiento y defenestración de un movimiento internacional a favor de reformas sexuales, con congresos de igual nombre, y el ascenso del fascismo, se pusieron de manifiesto las conexiones entre sexualidad y política. Por esos años Wilhem Reich teorizó sobre la tal conexión al asociar liberación sexual y liberación política, en la medida en que la primera debía producir una modificación del carácter de las personas y proporcionarles la energía que les permitiera sacudirse el yugo del autoritarismo (Reich, 1985). Pero la asociación entre sexualidad y política sólo dejó de ser una cuestión marginal en los años sesenta y setenta, a causa en buena medida del feminismo.

Aunque ya hemos mencionado a Foucault por la gran influencia que han tenido sus escritos a la hora de plantear un nuevo enfoque constructivista en el análisis de la sexualidad, Foucault no deja de ser deudor no confeso de las aportaciones feministas en torno a esta temática de la sexualidad y el poder, como muy bien señala Alicia Puleo en su libro La dialéctica de la sexualidad. Entre 
estas aportaciones destaca en los inicios de la segunda ola del feminismo la figura de Kate Millet con su obra Politica sexual. En este libro K. Millet sostiene que la sexualidad refleja la tensión entre la dominación masculina y la subordinacion femenina, idea ya conocida, como hemos mencionado hace un momento, pero que ella subrayó al destacar la importancia de las diferencias de poder en el terreno de la sexualidad. Millet se propuso demostrar que uel sexo es una categoría social impregnada de política", entendida ésta no en el sentido convencional que se manejaba por entonces de las reuniones, los presidentes y los partidos, sino como uel conjunto de relaciones y compromisos estructurados de acuerdo con el poder, en virtud de los cuales un grupo de personas queda bajo el control de otro grupo", en este caso las mujeres bajo el control de los hombres (Millet, 1975: 32).

Esta primera teoría sobre el patriarcado, enunciada en 1970 en el caldo de cultivo de un feminismo incipiente, dio paso en 1975 a la formulación de otra autora ya citada, Gayle Rubin, que escribió un artículo de gran influencia y que constituyó el arranque de una conceptualización que posteriormente el ferninismo utilizaría con profusión. El artículo se denominó "Tráfico de mujeres", término acuñado en tiempos por Emma Goldman para referirse a la prostitución. Rubin lo readopta sobre todo por sus implicaciones leviestrosianas en relación con las sociedades cuya organización se hallaba basada en el sistema de parentesco, en las que el intercambio de mujeres ocupaba un lugar destacado. En el antedicho trabajo Rubin acuña la terminología de "sistema de sexo/géneron - el sex-gender system-, que prefiere a la de patriarcado, a la que considera como más amplia y menos específica. Por «sistema de sexo/género" entiende uel conjunto de disposiciones por las que una sociedad transforma la sexualidad biológica en productos de la actividad humanas (Rubin, 1975: 159). Con este concepro Rubin pretendió dotarse, y dotar al ferninismo, de un instrumento conceptual que parecía describir adecuadamente «la organización social de la sexualidad" por medio, como ya hemos indicado, de una reinterpretación de Lévi-Strauss y su idea del intercambio de mujeres como base de la organización social. Nuestra autora pretendió explicar igualmente "la reproducción de las convenciones del sexo y del géneron a través de una relectura del complejo de Edipo freudiano, pasada por Lacan. La teoría freudiana, en cuanto que racionalización de la subordinación femenina, es rechazada por Rubin, quien la interpreta más bien como la descripción de un proceso que subordina a las mujeres y que, en tanto vista desde esta óptica, estima aprovechable por el feminismo.

No obstante la propia Gayle Rubin, en un escrito posterior de 1984 recogido en el libro preparado por Carole Vance titulado Placer y peligro, cuestionó su anterior formulación en el sentido de negarle al feminismo el privilegio de su enfoque a la hora de entender la subordinación sexual de las mujeres. Continúa considerando al feminismo como la teoría de la opresión de género, pero cuestiona su supremacía en cuanto teoria de la opresión sexual puesto que, según ella, asumir esto implica la ausencia de distinción entre el género, por un lado, y el sexo, entendido como el deseo erótico, por otro. Por 
supuesto que, sigue nuestra autora, el desarrollo del moderno sistema sexual - el que comienza, según Foucault, a partir de la era moderna, del siglo dieciocho- ha renido lugar en un contexto de opresión genérica; además, no caben dudas acerca de que el disfrute sexual ha sido concebido como un atributo y un privilegio masculinos, y de que el modelo de sexualidad vigente es un modelo que favorece al varón. Pero analíticamente convendría separarlos, indica Rubin, aunque ello suponga ir contra corriente de la mayoría del pensamiento feminista, que opina que la sexualidad se deriva del género.

Como ejemplo cita Rubin la opresión de las lesbianas: además de verse oprimidas como mujeres, son tachadas por añadidura de pervertidas o desviadas; lo que en este caso entra en juego es un sistema de estrarificación sexual, aplicable iguaimente a los hombres gays, los travestis y las prostitutas. Rubin tiene en cuenta lo muchísimo que en común poseen el sistema de género y el sistema sexual, pero aún así propone el desarrollo autónomo de una teoría y una política especificas a la sexualidad a fin de poder comprender y abarcar la organización social de la sexualidad. Esta es una propuesta que necesita mayor elaboración y reflexión pero que yo lanzo a la palestra en su formulación original, puesto que supone una interesante aportación que conviene no perder de vista.

Nos centraremos a continuación en algunas cuestiones relativas al enfoque construcrivista en torno a la sexualidad que aquí estamos manejando. Creo recordar que era Carole Vance la que expresaba gráficamente que "el principal órgano sexual se halla situado entre las orejasn. Ello da idea de la dimensión no natural y no esencial, en el sentido de principios inmutables, de la sexualidad, así como de la necesidad de entenderla como una construcción histórica y social, no basada meramente en la biología, aunque esto último sea, por supuesto, innegable. De todas formas resulta mucho más fácil, metodologicamente hablando, el pensar en términos de una libido natural que ha sido duramente reprimida, que la reflexión, cuando seguimos un modelo constuctivista, acerca de las injusticias en torno a la sexualidad (Rubin, 1984: 277). Como señala Carole Vance: «la teoría de la construcción social contiene en sí misma un problema para todo aquel que estudie la sexualidad, y es que te obliga a cuestionarte tus propias categorías y a darte cuenta de que son cultural e históricamente específicas". No podemos olvidar que el feminismo "participa en gran medida de este enfoque "constructivista", ya que su pregunta básica descansa sobre la forma en que está construido el "género", la forma en que se ha ido creando la noción de "mujer" (Osborne, 1990). Como escribe Ann Snitow, la misma conciencia de la "no naturalidad" de dicha noción contribuye a crear una tensión básicá dentro del ferminismo entre, por una parte, la necesidad de de-construir la categoría de mujer, por medio de la cual ha sido definida y encerrada en un estrecho marco de actuación; $y$, por otra, la necesidad de construir o de tomar conciencia de la propia identidad en tanto que mujeres y dotarla de un sólido significado político, imprescindible para construir un movimiento (Snitow, 1989: 205). 
En ta misma línea (que Snitow), Vance cree que hay un genuino problema: el planteamiento que desde esta perspectiva se establece sobre el cuerpo. Resulta obvio que un enfoque de esta índole rechaza las teorías deterministas de corte biologista o esencialista, pero también ha habido una tendencia a actuar como si el cuerpo no existiera. Así que aquí nos enfrentamos con una nueva tensión: por una parte parece arriesgado hacer casi desaparecer el cuerpo en la teoría de una manera que no es realmente plausible en relación con nuestra propia experiencia, pero si por otra parte queremos incluirlo, ¿cómo podemos hacerlo sin volver a caer en posturas esencialistas? se pregunta nuestra autora. Porque lo que está dando la impresión en los debates feministas es de que se está generando un cierto hiperdesarrollo de las teorías sobre la sexualidad sin contraste con una base empírica suficiente, con datos acerca de la realidad sexual de las mujeres ( $\mathrm{y}$ los hombres). A menudo, señala Vance, las teóricas feministas que escriben sobre la sexualidad lo hacen sobre una base insuficiente de datos o como si hubiera una experiencia femenina universal. $Y$ si bien esto ha sucedido tradicionalmente en el campo de la sexualidad mientras sólo los hombres controlaban esta disciplina, y aun así esta tendencia comenzó a corregirse en un momento dado, quizás ahora las feministas sigan cometiendo, en parte, el mismo error.

Esto.sucede, se apunta, entre otras, por dos razones: a) Porque las feministas no están muy familiarizadas con la sexología, la cual, a pesar de todos sus problemas de sexismo y de ciertos presupuestos que da por sentados acerca de las mujeres, ha recogido un montón de datos empíricos que muestran una gran diversidad en las formas de la sexualidad de estas últimas. b) Otra causa puede deberse a la ausencia de investigaciones sobre la sexualidad, o la sexualidad femenina si se quiere, efectuadas con un enfoque constructivista que haya hecho especial hincapié en la perspectiva de género (Osborne, 1990). Cuando esto se logre realizar, y éste parece un foro apropiado para comentar la necesidad de tales investigaciones, se podría quizás dejar de pontificar sobre "las experiencias sexuales de las mujeres", así como de transformar nuestras propias creencias en supuestos «datos» relativos a los peligros o los deseos de las mujeres.

Siguiendo a Foucault, Weeks y Rubin fundamentalmente, habremos de contemplar las numerosas fuerzas que intervienen en la conformación de la sexualidad - la economía, la política, la religión o las grandes ideologías como el psicoanálisis, los sistemas educativo, legal y sanitario-y su imbricación con instituciones "privadas" como pueder ser el matrimonio, la familia, el cuidado de los hijos, el hogar, la intimidad y el amor. Un análisis político acerca del sexo sólo es posible si éste no es concebido exclusivamente como un fenómeno biológico o como un aspecto de la psicología individual (Rubin, 1984: 275). La dimensión histórica y social de la sexualidad permite tener en cuenta cómo influyen en ella fenómenos relacionados con la demografía, la inmigración, los conflictos urbanos, cuestiones epidemiológicas - $\tan$ manifiestas ahora con la cuestión del sida_, etc. Es mucho más fructífero y menos prejuicioso a la hora de establecer las políticas sexuales tener en cuenta estos factores que los tradi- 
cionales criterios de pecado, enfermedad, patología, neurosis o decadencias que a todas/os suenan familiares en relación con la forma de enjuiciar la sexualidad (ibidem: 276).

Resulta evidente que la sexualidad ocupa un muy importante lugar tanto en nuestra opresión como en nuestro proyecto de liberación. Son las dos vertientes tan bien sintetizadas en el rótulo de "Placer y peligro" con que titula su libro Carole Vance. El movimiento de mujeres ha luchado denodadamente por poner en primer plano de las conciencias y de las legislaciones los problemas relativos a las agresiones sexuales y a la reproducción -incluidos la anticoncepción, el aborto y las nuevas tecnologías reproductivas -; ha denunciado el modelo masculino de sexualidad, que promueve mores privados y unas formas públicas - como por ejemplo la pornograffa y la prostituciónque privilegian una sexualidad hecha por y para el varón; ha criticado igualmente la invisibilidad de las lesbianas. Pero queda mucho por hacer, y por lo pronto y ya que estamos aquí en este contexto, convendría resaltar la conveniencia de tratar estos asuntos desde la Academia con el rigor que se merecen. Sólo si nos dotamos de instrumentos metodológicos eficaces lograremos que sean tomados en serio al tiempo que conseguiremos avanzar en el conocimiento de una materia que nos afecta a todas y a todos. Muchas gracias.

\section{Bibliografía}

FoucAult, Michel (1978). Historia de la sexualidad. Vol. I: "La voluntad de saber" (trad. Ulises Guiñazú). Madrid: Siglo XXI (ed. original, 1976).

Millet, Kate (1975). Politica sexual. (Trad. Ana María Bravo García). México D.F.: Aguilar (ed. original, 1969).

Osborne, Raquel (1990). "La sexualidad, una laguna del feminismo. (Entrevista a Carole Vance)". Hoja informativa del Colegio Nacional de Doctores y Licenciados en Ciencias Politicas y Sociologia, febrero.

Puleo, Alicia (1992). La dialéctica de la sexualidad. Género y sexo en la filosofia contemporánea. Madrid: Cátedra.

RubiN, Gayle (1989). "Reflexionando sobre el sexo: notas para una teoría radical de la sexualidad", en Carole Vance (comp.) Placer y peligro: explorando la sexualidad femenina, (trads. Julio Velasco y $\mathrm{M}^{\mathbf{a}}$ Ángeles Toda). Madrid: Revolución, p. 113-190. (Ed. original, 1984).

- (1975). "The Traffic in Women: Notes on the "Political Economy" of Sex", en Rayna R. Reiter (ed.) Toward an Anthropology of Women. Nueva York y Londres: Monthly Review Ptess, p. 175-210.

SCHuR, Edwin M. (1980). The Politics of Deviance. Stigma Contests and the Uses of Power, Englewood Cliffs, N. J.: Prentice Hall.

WeEKS, Jeffrey (1985). Civilization and its Discontents. Meanings, Myths and Modern Sexualities. Londres y Boston: Routledge and Kegan Paul Versión española: $E l$ malestar de la sexualidad. Significados, mitos y sexualidades modernas, trad. Alberto Magner. Madrid, Talasa, 1993).

- (1986). Sexuality. Chichester y Londres: Ellis Horwood/Tavistock. 